\title{
Implementation of An Automatic Fingerprint Identification System
}

\author{
Peihao Huang, Chia-Yung Chang and Chaur-Chin Chen * \\ Department of Computer Science \\ National Tsing Hua University \\ Hsinchu 30013, Taiwan \\ Email:cchen@cs.nthu.edu.tw
}

\begin{abstract}
Fingerprints used for identification and verification has attracted many biometric researchers. Although no identical fingerprint from distinct identities was found, a perfect automatic fingerprint identification system (AFIS) may not exist. This paper implements an AFIS with the use of fingerprint classification and a minutiae pattern matching. Our system is tested on a database composed of 112 $300 \times 300$ right index fingerprint images contributed by 28 persons, each has 4 duplicates via Veridicom FPS110 reader. Each match takes 0.2 seconds on a PC with Athelon XP 2500 $C P U$ and 512 MB SDRAM running Windows XP. We achieve 110 correct matches out of 112 tests. The ony two mismatched images are due to unavoidable noise. The result of testing our system on $4 \times 101$ left index fingerprints of more noisy images from 101 persons achieves $88 \%$ recognition rate.
\end{abstract}

Index Terms-bifurcation, core, delta, ending, minutiae pattern.

\section{Introduction}

Fingerprint used for access control, criminal verification, credit card and passport authentication, and digital right management is getting more and more popular. Whereas, a perfect automatic fingerprint identification system (AFIS) has not been discovered yet [6] due to an unexpected variety of changes when a fingerprint is sensed [6][10]. This paper implements an AFIS which receives a fingerprint image as input to match previously registered ones in the database and responds either "accept" or "reject" according to a designer-specified policy [3][5][7]. Our paradigm of minutiae-based AFIS is shown in Figure 1. The remaining of this paper depicts the details of this paradigm and shows intermediate image results of our procedures.

\footnotetext{
*This work is supported by Taiwan NSC 95-2221-E-007-198.
}

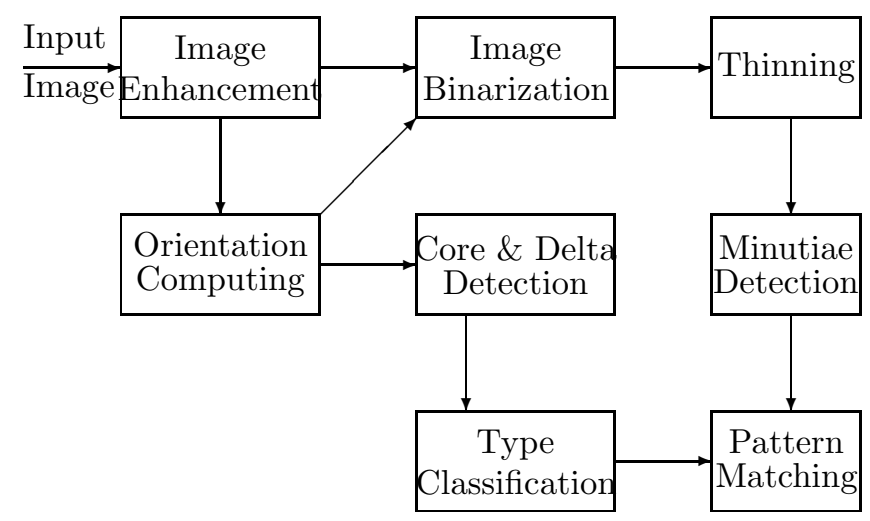

Figure 1. A System of Fingerprint Identification.

\section{Image Enhancement}

Our AFIS takes a fingerprint image of size $300 \times 300$ with 256 possible gray levels of resolution $500 \mathrm{dpi}$ as input. Figure 2(a) shows an example. Although a live scan technique was investigated to improve the quality of acquired fingerprint images, the moisture and scars of a finger as well as the pressure due to a fingerprint sensing could distort the quality of the acquired fingerprint image. We adopt an ad hoc strategy according to previous studies to enhance the quality of a fingerprint image.

Suppose that $A(i, j)$ is the image gray level at pixel $(i, j)$, we adopt the following transformation to stretch the distribution of gray levels [3][7].

$$
B(i, j) \leftarrow \alpha+\gamma *([A(i, j)-u] / s),
$$

where $u$ and $s^{2}$ are the mean and variance of gray levels of the input image, respectively, and $\alpha=150$, $\gamma=95$ were used [3]. Note that the selection of $\gamma$ must satisfy $\gamma>s$. Figure 2(b) shows the enhanced image by contrast stretching. 


\section{Image Binarization}

A minutiae pattern of a fingerprint image consists mainly of ridge endings and bifurcations associated with ridge orientations. To extract a minutiae pattern, binarization is important. Image binarization classifies each pixel into either "ridge" (darker) or "valley" (lighter). From an enhanced image $\{B(i, j)\}$ in Figure 2(b), we apply a binarization operation according to the following rules:

(1) Assign "ridge" to pixel $(i, j)$ if $B(i, j) \leq P_{k}$, where $P_{k}$ is the kth percentile of histogram of $\{B(i, j)\}$, e.g., $\mathrm{k}=25$.

(2) Assign "valley" to pixel $(i, j)$ if $B(i, j) \geq P_{50}$.

(3) The remaining pixels $\{(i, j)\}$ are classified as "valley" if $B(i, j) \geq T_{5 \times 5}$, and "ridge" otherwise; where $T_{5 \times 5}$ is the 30 th percentile of $5 \times 5$ pixel values surrounding the pixel $(i, j)$.

A result of binarization is shown in Figure 2(c).

\section{Skeletonization}

After binarization, a binary image is comprised of ridge and valley pixels. The width of a ridge at this step is in average 8 12 (pixels) which remains some difficulties to overcome for extracting the minutia points. The process of skeletonization is an iterative thinning processes which reduces a binary image consisting of ridges and valleys into a ridge map of unit width. Figure 2(d) shows the result by using a thinning algorithm proposed by Naccache and Singhal [9].

\section{Minutiae Pattern Detection}

From a thinned image, we can classify each ridge pixel into one of the following categories according to its 8connected neighbors. A ridge pixel is called

- an isolated point if it does not have any 8-connected neighbor,

- an ending if it has exactly one 8-connected neighbor,

- an edgepoint if it has two 8-connected neighbors,

- a bifurcation if it has three 8-connected neighbors, and

- a crossing if it has four 8-connected neighbors.
Two kinds of minutia pixels, either ending or bifurcation, are first located by checking the 8 neighbors of a ridge pixel. Figure 2(e) shows candidates of minutia pixels in a ridge map. Due to broken ridges, hair effects, and ridge endings beyond the margins, a postprocessing [3] is used to remove the following spurious minutia pixels with the result shown in Figure 2(f).

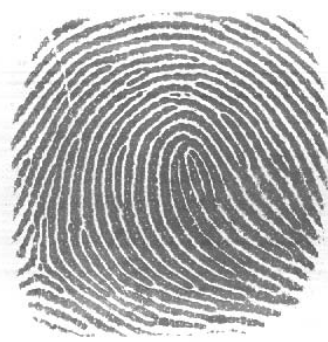

(a)

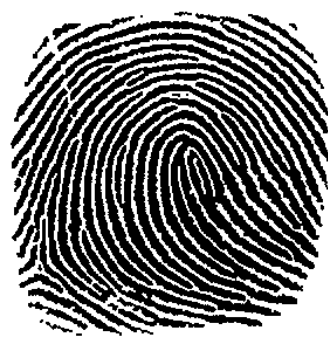

(c)

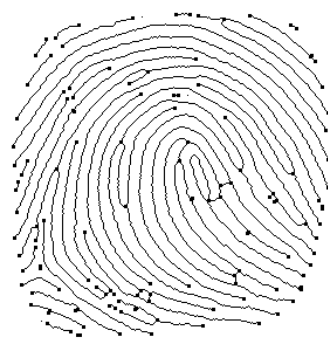

(e)

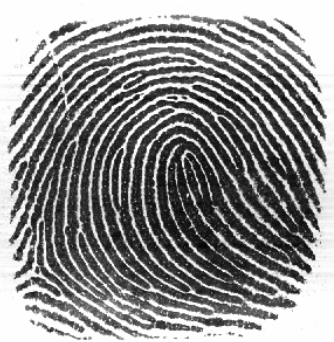

(b)

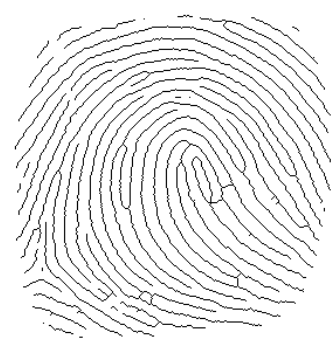

(d)

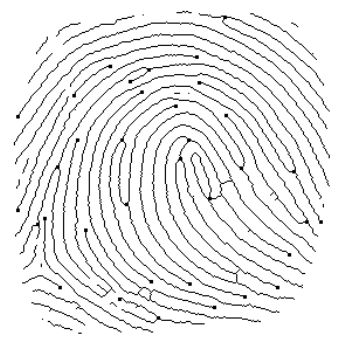

(f)
Figure 2. Minutiae Extraction: (a) Original image, (b) Contrast Stretching, (c) Binarization, (d) Skeletonization, (e) Minutia Points, (f) Minutiae Pattern.

\section{A. Minutiae Pattern}

Spurious minutia pixels include (1) endings that lie on the margins of the region of interest as shown in Figure 3(a), (2) two closest endings of the same ridge orientation which are broken as shown in Figure 3(b), (3) ending and bifurcation that are connected and close enough as shown in Figure 3(c), and (4) two bifurcations that are too close as shown in Figure 3(d). The closeness here is defined as 8 pixels wide. 
IEEE EIT 2007 Proceedings

After removing the spurious minutia pixels, a minutiae pattern, consisting of approximately $20 \sim 40$ ridge endings and bifurcations associated with corresponding ridge orientations, is obtained for matching pre-stored minutiae patterns in a database.

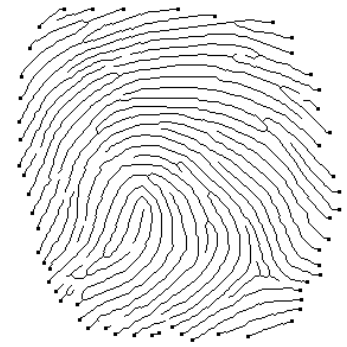

(a)

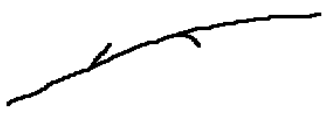

(c)

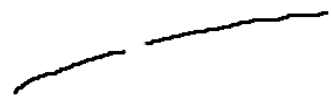

(b)

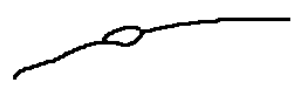

(d)
Figure 3. False Minutiae: (a) Beyond margins, (b) Broken ridges, (c) Hair effect, (d) Too close bifurcations.

\section{Type Classification of Fingerprints}

Since a minutiae-based pattern matching procedure in a large database may consume a huge amount of time, we classify each fingerprint into one of the four types: left loop, right loop, whorl, and arch, and search in the database only for those fingerprints whose type coincides with that of a requested one. The type classification is based on the number and distribution of singular points: core and deltas [4][2][8][11][12] which are described in the following subsections.

\section{A. Ridge Orientation Map}

A pixel orientation map is computed according to the reports of [1][3][5]. For pixel $(i, j)$, we apply Sobel operators to get

$$
\begin{aligned}
G_{x}= & B(i+1, j-1)+2 B(i+1, j)+B(i+1, j+1) \\
& -B(i-1, j-1)-2 B(i-1, j)-B(i-1, j+1) \\
G_{y}= & B(i-1, j+1)+2 B(i, j+1)+B(i+1, j+1) \\
& -B(i-1, j-1)-2 B(i, j-1)-B(i+1, j-1)
\end{aligned}
$$

$$
\rho=\sqrt{G_{x}^{2}+G_{y}^{2}}, \quad \phi=\tan ^{-1}\left(G_{y} / G_{x}\right),
$$

where $\phi$ could be regarded as pixel orientation for $(i, j)$. To further compute the ridge orientation, let

$$
\begin{gathered}
\alpha_{x}=G_{x}^{2}-G_{y}^{2}, \quad \alpha_{y}=2 G_{x} G_{y}, \\
\beta_{x}=\sum_{R} G_{x}^{2}-G_{y}^{2}, \quad \beta_{y}=\sum_{R} 2 G_{x} G_{y}
\end{gathered}
$$

The block direction for region $R(11 \times 11$ block $)$ centered at pixel $(i, j)$ can be computed by $\Phi=\frac{1}{2} \theta$, where

$$
\theta= \begin{cases}\tan ^{-1}\left(\beta_{y} / \beta_{x}\right) & \text { if } \beta_{x} \geq 0 \\ \tan ^{-1}\left(\beta_{y} / \beta_{x}\right)+\pi & \text { if } \beta_{x}<0 \text { and } \beta_{y} \geq 0 \\ \tan ^{-1}\left(\beta_{y} / \beta_{x}\right)-\pi & \text { if } \beta_{x}<0 \text { and } \beta_{y}<0\end{cases}
$$

Apply a $3 \times 3$ median filter followed by quantization (into 8 directions) on $\left\{\Phi^{\prime} s\right\}$, we have a map of ridge orientation as shown in Figure 4.

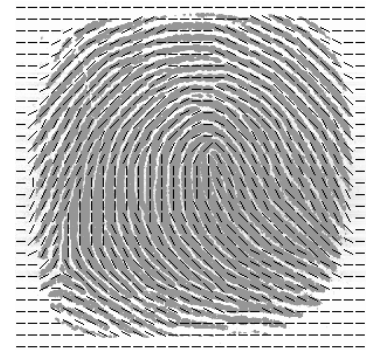

(a)

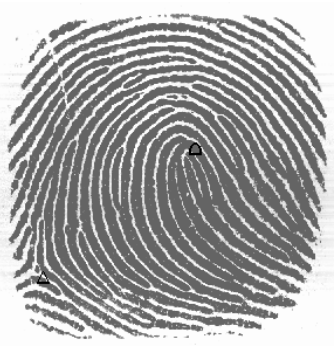

(b)
Figure 4. (a) Map of Ridge Orientation, (b) Core and Delta.

A Poincaré index as defined in [5] was adopted for finding the cores and deltas. A left loop might contain a delta located on the right and/or down of the core which is convex. A right loop might contain a delta located on the left and/or down of the core which is convex. A whorl contains cores without deltas in the surroundings. An arch has a delta almost right below the core. This coarse classification can significantly prune mismatched comparisons and speed up the minutiae patern matching.

Minutiae pattern matching is an important but difficult task. The performance is quite different from one database to another database. We adopt the strategy 
IEEE EIT 2007 Proceedings

proposed by Chen and Wang [2]. Given a test pattern $P$, we search from the database with the same type, say, a $Q$ to compute a matching score, the larger, the better match. The matching score is calculated by

$$
S=\frac{1}{2 M} \sum_{j=1}^{M}\left[\left(1-\frac{r_{j}}{R}\right)+\left(1-\frac{\alpha_{j}}{A}\right)\right]
$$

where $\mathrm{M}$ is the number of potential type-matching minutiae (pixels) within a disk of a certain userspecified radius, $R$, under a rotation with a certain angle $\alpha_{j}$ (here $A=\pi$ ). Each $r_{j}$ measures the distance between a pair of potentially matched minutia points with $\alpha_{j}$ measuring the difference of orientations.

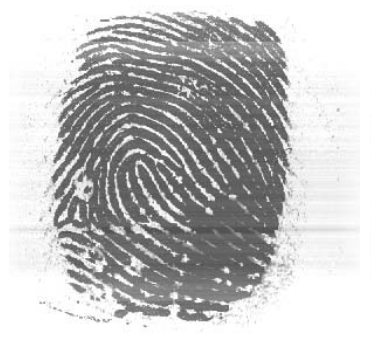

(a)

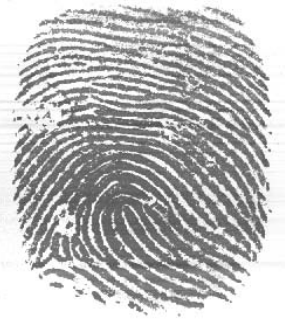

(b)
Figure 5. (a) Image of much moisture, (b) Image of many scars.

\section{Experiment and Discussion}

We tested our proposed AFIS on a database consisting of 112 right index fingerprint images of size $300 \times 300$ which were contributed from 28 individuals, each contributed 4 times of his or her right index fingerprint with a pre-attention on impressing their fingerprints on the surface of the reader almost at a fixed spot via a Veridicom FPS110 reader. So only a slight of rotation and translation from one fingerprint to another may exist for each person. To evaluate our AFIS, one fingerprint image is matched with the remaining 111 images in the database, our AFIS achieves the highest score for 110 images out of 112 total fingerprint images. The only two mismatched ones come from the same person suspected to have much moisture and many scars on his/her fingerprint surface as shown in Figures $5(\mathrm{a} \sim \mathrm{b})$. The result of testing our system on $4 \times 101$ left index fingerprints of more noisy images from 101 persons (Database Lindex101) achieves 88\% recognition rate. Our system adopts a coarse fingerprint classification for minutiae pattern matching. It takes around 0.2 seconds for each identification. Other techniques of enhancement and minutiae matching strategies [8][11] to improve the performance are under investigation.

\section{References}

[1] R. Cappelli, A. Lumini, D. Maio, D. Maltoni, Fingerprint Classification by Directional Image Partitioning. IEEE Trans. on Pattern Analysis and Machine Intelligence Vol. 21, 402-421, 1999.

[2] C.C. Chen and Y.Y. Wang, An AFIS Using Fingerprint Classification, Image and Vision Computing New Zealand Conference, New Zealand, 233-238, 2003.

[3] C.Y, Chang, Automatic Fingerprint Verification System. M.S. Thesis, National Tsing Hua University, Taiwan, June 2003.

[4] The Science of Fingerprints: Classification and Uses, U.S. Department of Justice, Federal Bureau of Investigation, Washington, rev. 12-84, 1999.

[5] A.K. Jain, L. Hong, S. Pankanti, R. Bolle, An Identity-Authentication System Using Fingerprints. Proceedings of The IEEE, Vol. 85, No. 9, 1364-1388, 1997.

[6] A.K. Jain, S. Pankanti, S. Prabhakar, L. Hong, and A. Ross, Biometrics: A Grand Challenge, International Conference on Pattern Recognition, Cambridge, England, 2004.

[7] P. Huang, Implementation for AFIS, M.S. Thesis, National Tsing Hua University, Taiwan, March 2004.

[8] D. Mario, R. Cappelli, J.L. Wayman, and A.K. Jain, FVC2000: Fingerprint Verification Competition. IEEE Trans. on Pattern Analysis and Machine Intelligence, Vol. 24, No. 3, 402-412, 2002.

[9] N.J. Naccache and R. Shinghal, An investigation into the skeletonization approach of Hilditch. Pattern Recognition, Vol. 17, 279-284, 1984.

[10] S. Prabhakar, A.K. Jain, S. Pankanti, R. Bolle, Minutia Verification and Classification for Fingerprint Matching. Proceedings of the 15th Int'l Conference on Pattern Recognition, Barcelona, Spain, Vol. 1, 25-29, 2000.

[11] A. Ross, A.K. Jain, and J. Reisman, A Hybrid Fingerprint Matcher, Pattern Recognition, Vol. 36, No. 7, 1657-1672, 2004.

[12] M. Tico and P. Kuosmanen, Fingerprint matching Using an Orientation-Based Minutia Descriptors, IEEE Trans. on Pattern Analysis and Machine Intelligence, Vol. 25, No. 8, 1009-1014, 2003. 